\title{
2PI functional techniques for abelian gauge theories
}

\author{
U. Reinosa ${ }^{a}$ and J. Serreau ${ }^{b}$ \\ ${ }^{a}$ Centre de Physique Théorique, École Polytechnique, 91128 Palaiseau, France ${ }^{1}$ \\ ${ }^{\mathrm{b}}$ Astro-Particule et Cosmologie, Université Paris 7 - Denis Diderot, \\ 10, rue A. Domon et L. Duquet, 75205 Paris Cedex 13, France ${ }^{2}$
}

\begin{abstract}
We summarize our recent work $[1,2,3]$ concerning the formulation of two-particle-irreducible (2PI) functional techniques for abelian gauge field theories.

Key words: Gauge theories, Nonperturbative methods, 2PI effective action, Renormalization PACS: 11.15.-q, 11.15.Tk, 12.38.Cy, 11.10.Gh
\end{abstract}

\section{Introduction}

Two-particle-irreducible (2PI) functional techniques provide a powerful tool to devise systematic nonperturbative approximation schemes in quantum field theory, of interest in numerous physical situations where standard (loop, $1 / N$ ) expansions fail. A non-trivial issue for such approximations is that they should reflect, as much as possible, basic features of the exact theory, such as symmetries and renormalizability.

The issue of renormalization in the $2 \mathrm{PI}$ formalism has attracted a lot of attention in recent years 456178 , and is now a well established topic. The basic tools have been put forward in pioneering works [4] and a complete $2 \mathrm{PI}$ renormalization theory has been proposed for theories with linearly realized global symmetries [5].

In a recent series of papers [1/2]3, we have developed the 2PI renormalization theory for abelian gauge theories. This requires a proper understanding of symmetry constraints, i.e. Ward-Takahashi (WT) identities, in the 2PI formalism, which is the purpose of Ref. 11. In Refs. 23, these results are used to develop a consistent renormalization procedure for 2PI QED, which preserves the underlying gauge-symmetry. The present contribution provides a summarized account of these papers.

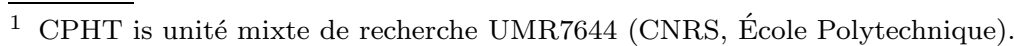

2 APC is unité mixte de recherche UMR7164 (CNRS, Université Paris 7, CEA, Observatoire de Paris). 


\section{2PI effective action for QED}

We consider QED in the covariant gauge and use dimensional regularization. The gauge-fixed classical action reads, with standard notations,

$$
S[A, \psi, \bar{\psi}]=\int d^{d} x\left\{\bar{\psi}[i \not \partial-e A-m] \psi+\frac{1}{2} A^{\mu}\left[g_{\mu \nu} \partial^{2}-(1-\lambda) \partial_{\mu} \partial_{\nu}\right] A^{\nu}\right\},
$$

where $\lambda$ is the gauge-fixing parameter. Aside from the gauge-fixing term, the classical action is invariant under the gauge transformation

$$
\psi(x) \rightarrow e^{i \alpha(x)} \psi(x), \quad \bar{\psi}(x) \rightarrow e^{-i \alpha(x)} \bar{\psi}(x), \quad A_{\mu}(x) \rightarrow A_{\mu}(x)-\frac{1}{e} \partial_{\mu} \alpha(x),
$$

where $\alpha(x)$ is an arbitrary real function. To define the $2 \mathrm{PI}$ effective action, it is convenient to grab the bosonic and fermionic connected one- and two-point functions in a superfield $\varphi$ and corresponding supercorrelator $\mathcal{G}$ (transposition includes space-time variables):

$$
\varphi=\left(\begin{array}{c}
A \\
\psi \\
\bar{\psi}^{t}
\end{array}\right), \quad \mathcal{G}=\left(\begin{array}{ccc}
G & K^{t} & \bar{K} \\
K & F & D \\
\bar{K}^{t} & -D^{t} & \bar{F}
\end{array}\right) .
$$

In general, one should keep track, at the level of the 2PI effective action, of all possible fields and correlators. Besides the usual photon and fermion two-point functions $G$ and $D$, this includes all possible mixed correlators, such as, for instance the photon-fermion correlators $K$ and $\bar{K}$, etc. Although the latter vanish on-shell, they should be set to their physical value only after all the relevant functional derivatives have been taken 3

Writing the classical action as $S[\varphi]=S_{0}[\varphi]+S_{\text {int }}[\varphi]$, where $S_{0}[\varphi]=\frac{1}{2} \varphi_{m} i \mathcal{G}_{0, m n}^{-1} \varphi_{n}$ is the quadratic part, with $\mathcal{G}_{0}^{-1}$ the free inverse (super)propagator, the $2 \mathrm{PI}$ functional can be parametrized as [19]:

$$
\Gamma_{2 \mathrm{PI}}[\varphi, \mathcal{G}]=S_{0}[\varphi]+\frac{i}{2} \operatorname{Str} \operatorname{Ln} \mathcal{G}^{-1}+\frac{i}{2} \operatorname{Str} \mathcal{G}_{0}^{-1} \mathcal{G}+\Gamma_{\mathrm{int}}[\varphi, \mathcal{G}]
$$

where Str denotes the functional supertrace and $i \Gamma_{\text {int }}[\varphi, \mathcal{G}]$ is the sum of closed twoparticle-irreducible (2PI) diagrams made of classical QED vertices and lines given by $\mathcal{G}$.

The physical correlator $\overline{\mathcal{G}}[\varphi]$ in the presence of a nonvanishing field $\varphi$ is obtained as the solution of the stationarity condition $\delta \Gamma_{2 \mathrm{PI}} / \delta \mathcal{G}=0.4$.

$$
\overline{\mathcal{G}}^{-1}[\varphi]=\mathcal{G}_{0}^{-1}-\bar{\Sigma}[\varphi] \quad \text { with }\left.\quad \bar{\Sigma}_{m n}[\varphi] \equiv(-1)^{q_{n}} 2 i \frac{\delta \Gamma_{\mathrm{int}}[\varphi, \mathcal{G}]}{\delta \mathcal{G}_{n m}}\right|_{\overline{\mathcal{G}}[\varphi]}
$$

Finally, the effective action $\Gamma[\varphi]$, the generating functional for 1PI $n$-point vertex functions, is obtained as

$$
\Gamma[\varphi] \equiv \Gamma_{2 \mathrm{PI}}[\varphi, \overline{\mathcal{G}}[\varphi]]
$$

3 For instance, $\delta \Gamma_{2 \mathrm{PI}} / \delta K \delta \bar{K}$ does not vanish on-shell.

4 Here, $(-1)^{q_{n}}=1$ if $n$ refers to a bosonic superfield component and $(-1)^{q_{n}}=-1$ otherwise. 
This is a mere identity in the exact theory. However, for finite 2PI approximations, Eqs (44)-(6) define a powerful systematic resummation scheme for the effective action 513 .

\section{Vertex functions}

In the $2 \mathrm{PI}$ framework, $n$-point vertex functions can be obtained in different ways, see e.g. 5]. Equivalent in the exact theory, they differ in general at finite approximation order. The most straightforward definition involves the $n$-th derivatives of the (2PI-resummed) effective action (6):

$$
\left.\Gamma_{1 \ldots n}^{(n)} \equiv \frac{\delta^{n} \Gamma[\varphi]}{\delta \varphi_{n} \cdots \delta \varphi_{1}}\right|_{\bar{\varphi}}
$$

taken at the physical value $\bar{\varphi}$ of the field, defined by $\delta \Gamma[\varphi] /\left.\delta \varphi\right|_{\bar{\varphi}}=0$. We refer to these as 2PI-resummed vertex functions.

Other possible definitions of vertex functions involve derivatives of the 2PI effective action (44) with respect to $\mathcal{G}$. For instance, the two-point function - the self-energy - can either be obtained from the second derivative of the 2PI-resummed effective action, see Eq. (7), or directly from Eq. (5). In turn, higher $n$-point functions can be obtained as field-derivatives of the self-energy $\bar{\Sigma}[\varphi]$ :

$$
\left.i V_{m n ; 1 \cdots p}^{(p+2)} \equiv(-1)^{q_{m}} \frac{\delta^{p} \bar{\Sigma}_{n m}[\varphi]}{\delta \varphi_{p} \cdots \delta \varphi_{1}}\right|_{\bar{\varphi}} .
$$

Of course, $\Gamma^{(n)}=V^{(n)}$ in the exact theory [1, but this is not true in general at finite

approximation order. We refer to the vertices (8) as 2PI vertex function 5 . Together with 2PI-resummed vertices (7), they play a crucial role in the renormalization program.

\section{Symmetries and 2PI Ward-Takahashi identities [1]}

To analyze the role played by the gauge symmetry in the quantum theory, we write

$$
S[\varphi]=S_{\mathrm{sym}}[\varphi]+S_{\mathrm{gf}}[\varphi]
$$

where $S_{\mathrm{sym}}[\varphi]$ is the gauge-invariant classical QED action and $S_{\mathrm{gf}}[\varphi]$ is the gauge-fixing term. We consider the linear gauge transformation of the fields

$$
\varphi \rightarrow \varphi^{(\alpha)}=\mathcal{A} \varphi+\mathcal{B} \text { and } \mathcal{G} \rightarrow \mathcal{G}^{(\alpha)}=\mathcal{A} \mathcal{G} \mathcal{A}^{t},
$$

where we use the notation $\mathcal{A} \varphi \equiv \int_{y} \sum_{n} \mathcal{A}_{m n}(x, y) \varphi_{n}(y)$. For the transformation (2), one has $\mathcal{A}_{m n}(x, y)=\delta_{m n} \delta^{(4)}(x-y) \exp \left[i q_{m} \alpha(x)\right]$ and $\mathcal{B}(x)=(-\partial \alpha(x) / e, 0,0)^{t}$.

We show in Ref. [1] that, for linear gauges, the $2 \mathrm{PI}$ effective action must be of the form

$$
\Gamma_{2 \mathrm{PI}}[\varphi, \mathcal{G}]=\Gamma_{2 \mathrm{PI}}^{\mathrm{sym}}[\varphi, \mathcal{G}]+S_{\mathrm{gf}}[\varphi],
$$

where $\Gamma_{2 \mathrm{PI}}^{\mathrm{sym}}[\varphi, \mathcal{G}]$ is invariant under the gauge transformation (10). This generalizes the standard result that, for linear gauges, $S_{\mathrm{gf}}[\varphi]$ does not receive any loop corrections.

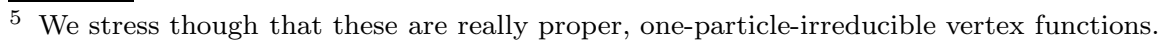


Equation (11) encodes all the symmetry identities of the quantum theory. For instance, since the gauge-fixing term in Eq. (11) is $\mathcal{G}$-independent, one concludes that the physical correlator $\overline{\mathcal{G}}[\varphi]$ is obtained as the extremum of the symmetric functional $\Gamma_{2 \mathrm{PI}}^{\mathrm{sym}}[\varphi, \mathcal{G}]$. It follows that it transforms covariantly under the gauge-transformation of its argument: $\overline{\mathcal{G}}\left[\varphi^{(\alpha)}\right]=\overline{\mathcal{G}}^{(\alpha)}[\varphi]$. For an infinitesimal transformation, this can be rewritten as (denoting by $\delta^{(\alpha)}$ the corresponding variations)

$$
\delta^{(\alpha)} \varphi_{p} \frac{\delta \bar{\Sigma}_{m n}[\varphi]}{\delta \varphi_{p}}=-\delta^{(\alpha)} \overline{\mathcal{G}}_{m n}^{-1}[\varphi] .
$$

Eq. (12) generates, through functional derivatives, a hierarchy of symmetry identities for the $2 \mathrm{PI}$ vertex functions (8). It is remarkable that, despite the rather unusual definition of the latter, the obtained identities have the very same form as the usual WT identities. As an illustration, the $(\bar{\psi}, \psi)$-component of Eq. (12) leads to the usual relation between three-point photon-fermion vertex function $\delta \bar{\Sigma}_{\bar{\psi} \psi} /\left.\delta A^{\mu}\right|_{\bar{\varphi}}$ and the inverse fermion twopoint function $i \bar{D}^{-1}$ :

$$
-\left.\frac{1}{e} \partial_{z}^{\mu} \frac{\delta \bar{\Sigma}_{\bar{\psi} \psi}(x, y)}{\delta A^{\mu}(z)}\right|_{\bar{\varphi}}=\left[\delta^{(4)}(x-z)-\delta^{(4)}(z-y)\right] i \bar{D}^{-1}(x, y) .
$$

Similarly, one obtains from the $(A, A)$-component of Eq. (12) that 2PI $n$-photon vertex functions with $n \geq 3$ are transverse in momentum space with respect to external momenta associated with field derivatives (see [1] for details):

$$
\left.\partial_{z_{1}}^{\mu_{1}} \frac{\delta \bar{\Sigma}_{A A}^{\rho \sigma}(x, y)}{\delta A^{\mu_{1}}\left(z_{1}\right) \cdots \delta A^{\mu_{k}}\left(z_{k}\right)}\right|_{\bar{\varphi}}=0 .
$$

Next, using Eq. (5), one finds that the 2PI-resummed effective action reads

$$
\Gamma[\varphi]=\Gamma^{\mathrm{sym}}[\varphi]+S_{\mathrm{gf}}[\varphi],
$$

where $\Gamma_{\text {sym }}[\varphi]=\Gamma_{2 \mathrm{PI}}^{\mathrm{sym}}[\varphi, \overline{\mathcal{G}}[\varphi]]$ is invariant under the gauge transformation (10). It immediately follows that 2PI-resummed vertices satisfy the standard WT identities. It is remarkable that, although they are a priori very different objects, $2 \mathrm{PI}$ and $2 \mathrm{PI}$-resummed vertices with three external legs or more independently satisfy the same WT identities.

An interesting byproduct of this analysis is that the 2PI two-point function itself, $i \overline{\mathcal{G}}^{-1} \equiv i \overline{\mathcal{G}}^{-1}[\bar{\varphi}]$, is not constrained by the underlying symmetry. This is because it is defined as the solution of a stationarity condition, Eq. (5), and not as a field derivative of some functional. In general, only the latter are constrained by symmetry identities. For instance, the $2 \mathrm{PI}$ photon polarization tensor $\bar{\Pi}^{\mu \nu} \equiv \bar{\Sigma}_{A A}^{\mu \nu}[\bar{\varphi}]$ is not constrained to be transverse in momentum space at any finite approximation order 6 In contrast, the 2PI-resummed two-point function $\Gamma^{(2)} \equiv \delta^{2} \Gamma[\varphi] /\left.\delta \varphi^{2}\right|_{\bar{\varphi}}$, being defined as a geometrical object, is constrained in the usual way by the gauge symmetry. In particular, the 2PIresummed photon polarization tensor $\Pi_{\mu \nu} \equiv \delta^{2}\left(\Gamma[\varphi]-S_{0}[\varphi]\right) /\left.\delta A^{\nu} \delta A^{\mu}\right|_{\bar{\varphi}}$ is transverse in momentum space:

$$
\partial_{x}^{\mu} \Pi_{\mu \nu}(x, y)=0 .
$$

${ }^{6}$ Of course a non-vanishing longitudinal component of $\bar{\Pi}$ is a pure artifact of the approximation. For instance, for a systematic loop expansion, it is always of higher order than the approximation order [2]. 
It is important to realize that all the results derived above are direct consequences of the symmetry property (11). It follows that any 2PI approximation which respects this property leads to nonperturbative expressions for (2PI and 2PI-resummed) vertex functions which exactly satisfy (2PI) WT identities. In Ref. [1, we give general rules to construct such approximation schemes for general abelian gauge theories. In particular, we show that, in QED, the 2PI loop-expansion satisfies the symmetry constraint (11) at any approximation order.

\section{Renormalization 2,3}

We define the rescaled field and propagator using the matrix $Z \equiv \operatorname{diag}\left(Z_{3}, Z_{2}, Z_{2}\right)$ :

$$
\varphi_{R}=Z^{-1 / 2} \varphi \quad \text { and } \quad \mathcal{G}_{R}=Z^{-1 / 2} \mathcal{G} Z^{-1 / 2},
$$

as well as the usual renormalized parameters $Z_{0} m_{R}=Z_{2} m, Z_{1} e_{R}=Z_{2} Z_{3}^{1 / 2} e$ and $Z_{4} \lambda_{R}=$ $Z_{3} \lambda$. The renormalized $2 \mathrm{PI}$ effective action, defined as $\Gamma_{2 \mathrm{PI}}^{R}\left[\varphi_{R}, \mathcal{G}_{R}\right]=\Gamma_{2 \mathrm{PI}}[\varphi, \mathcal{G}]$, can be written as, up to a constant contribution:

$$
\Gamma_{2 \mathrm{PI}}^{R}\left[\varphi_{R}, \mathcal{G}_{R}\right]=S_{0, R}\left[\varphi_{R}\right]+\frac{i}{2} \operatorname{Str} \ln \mathcal{G}_{R}^{-1}+\frac{i}{2} \operatorname{Str} \mathcal{G}_{0, R}^{-1} \mathcal{G}_{R}+\Gamma_{\mathrm{int}}^{R}\left[\varphi_{R}, \mathcal{G}_{R}\right],
$$

where $S_{0, R}\left[\varphi_{R}\right] \equiv \frac{1}{2} \varphi_{R}^{t} i \mathcal{G}_{0, R}^{-1} \varphi_{R}$ with $\mathcal{G}_{0, R}^{-1}$ obtained from $\mathcal{G}_{0}^{-1}$ after $(m, \lambda)$ is changed to $\left(m_{R}, \lambda_{R}\right)$. Eq. (18) defines $\Gamma_{\text {int }}^{R}\left[\varphi_{R}, \mathcal{G}_{R}\right]$, which can be expressed in terms of renormalized parameters and counterterms $\delta Z_{i}=Z_{i}-1, i=0, \ldots, 4$ :

$$
\Gamma_{\text {int }}^{R}\left[\varphi_{R}, \mathcal{G}_{R}\right]=\Gamma_{\text {int }}\left[\varphi_{R}, \mathcal{G}_{R} ; e_{R}\right]+\delta \Gamma_{\text {int }}\left[\varphi_{R}, \mathcal{G}_{R}\right] .
$$

The first term on the RHS is obtained from $\Gamma_{\text {int }}[\varphi, \mathcal{G}]$ by replacing bare quantities by renormalized ones whereas $\delta \Gamma_{\text {int }}\left[\varphi_{R}, \mathcal{G}_{R}\right]$ accounts for all counterterm contributions. For a successful renormalization program, the latter should be such that the infinitely many UV-divergences appearing at any finite approximation order in the $2 \mathrm{PI}$ formalism can be canceled by adjusting a finite number of local counterterms consistent with the underlying symmetries, a non-trivial task obviously.

In Refs. [2]3, we show that this can indeed be achieved if one includes in $\delta \Gamma_{\text {int }}\left[\varphi_{R}, \mathcal{G}_{R}\right]$ all counterterms contributions allowed by power counting and (Lorentz, gauge, etc.) symmetries. In the 2PI framework, this may allow for new type of counterterms, which have no analog in the standard renormalization theory, simply because there are more possibilities to construct symmetry invariants with both $\varphi_{R}$ and $\mathcal{G}_{R}$ than with $\varphi_{R}$ alone. As an illustration, the most general counterterm contribution satisfying the above requirements at two-loop order can be written as 3,7

$$
\delta \Gamma_{\mathrm{int}}\left[\varphi_{R}, \mathcal{G}_{R}\right]=\delta S_{\mathrm{int}}\left[\varphi_{R}\right]+\delta \Gamma_{\mathrm{int}}^{1 \mathrm{loop}}\left[\varphi_{R}, \mathcal{G}_{R}\right]+\delta \Gamma_{2}\left[\mathcal{G}_{R}\right]
$$

with

$$
\begin{aligned}
\delta S_{\mathrm{int}}\left[\varphi_{R}\right] & =\int d^{d} x \frac{\delta Z_{3}}{2} A_{R}^{\mu}(x)\left(g_{\mu \nu} \partial_{x}^{2}-\partial_{\mu}^{x} \partial_{\nu}^{x}\right) A_{R}^{\nu}(x) \\
& +\int d^{d} x \bar{\psi}_{R}(x)\left(i \delta Z_{2} \not_{x}-\delta m-\delta Z_{1} e_{R} A_{R}(x)\right) \psi_{R}(x),
\end{aligned}
$$

\footnotetext{
7 Only Eq. 231) receives higher-loop contributions.
} 


$$
\begin{aligned}
\delta \Gamma_{\text {int }}^{1 \text { loop }}\left[\varphi_{R}, \mathcal{G}_{R}\right] & =\left.\int d^{d} x\left(\frac{\delta \bar{Z}_{3}}{2}\left(g_{\mu \nu} \partial_{x}^{2}-\partial_{\mu}^{x} \partial_{\nu}^{x}\right)+\frac{\delta \bar{M}^{2}}{2} g_{\mu \nu}+\frac{\delta \bar{\lambda}}{2} \partial_{\mu}^{x} \partial_{\nu}^{x}\right) G_{R}^{\mu \nu}(x, y)\right|_{y=x} \\
& -\left.\int d^{d} x \operatorname{tr}\left(i \delta \bar{Z}_{2} \partial_{x}-\delta \bar{m}-\delta \bar{Z}_{1} e_{R} A_{R}(x)\right) D_{R}(x, y)\right|_{y=x} \\
& -\int d^{d} x \delta \tilde{Z}_{1} e_{R}\left(\bar{\psi}_{R}(x) K_{R}(x, x)+\bar{K}_{R}(x, x) \psi_{R}(x)\right)
\end{aligned}
$$

and

$$
\delta \Gamma_{2}\left[\mathcal{G}_{R}\right]=\int d^{d} x\left(\frac{\delta \bar{g}_{1}}{8} G_{R \mu}^{\mu}(x, x) G_{R \nu}^{\nu}(x, x)+\frac{\delta \bar{g}_{2}}{4} G_{R}^{\mu \nu}(x, x) G_{\mu \nu}^{R}(x, x)\right) .
$$

A few remarks are in order here. First, notice that some terms, which would be allowed by power counting and global symmetries, such as $A_{R}^{2}$, or $\left(\partial A_{R}\right)^{2}$ in Eq. (21), or $A_{R}^{2} G_{R}$ in Eq. (22), are forbidden by the gauge symmetry. Correspondingly, as shown in [3, 2PI WT identities prevent the appearance of those UV divergences which would call for such counterterms. In contrast, since the photon correlator $G_{R}$ is invariant under the gauge transformation (10) terms of the form $G_{R}, \partial \partial G_{R}$ and $G_{R}^{2}$ are allowed, giving rise to corresponding counterterms $\delta \bar{M}^{2}, \delta \bar{\lambda}, \delta \bar{g}_{1}$ and $\delta \bar{g}_{2}$, which would be absent in the exact theory. As discussed in detail in 2 these counterterms actually serve to absorb divergences in the longitudinal (in momentum space) part of the inverse photon correlator $\bar{G}_{R}^{-1}$ which, as discussed previously, are not forbidden by 2PI WT identities. Notice though that, as already mentioned, such divergences are a pure artifact of the approximation. For a 2PI loop-expansion, they are systematically of higher order than the approximation order. So are the (divergent part of the) corresponding counterterms which, therefore, vanish as one approaches the exact theory.

Next, notice that gauge symmetry allows for a priori different independent $\delta Z$ 's, such as $\delta Z_{3}$ and $\delta \bar{Z}_{3}$, etc. This is a generic feature of $2 \mathrm{PI}$ renormalization theory, related to the existence of a priori different possible definitions of vertex functions. The latter come with a priori different UV divergences, to be absorbed in the different $\delta Z$ 's. Again, at a given order in the $2 \mathrm{PI}$ loop-expansion, these differences are systematically higher-order effects. Finally, the terms $\bar{\psi}_{R} \not \partial \psi_{R}, \bar{\psi}_{R} A_{R} \psi_{R}, \not \partial D_{R}$ and $A_{R} D_{R}$ are obviously related by the gauge symmetry and so are the counterterms $\delta Z_{1}, \delta Z_{2}, \delta \bar{Z}_{1}$ and $\delta \bar{Z}_{2}$. For instance, for a suitable definition of the renormalized charge $e_{R}$, one has $\delta Z_{1}=\delta Z_{2}$ and $\delta \bar{Z}_{1}=\delta \bar{Z}_{2}$.

In Refs. 2[3] we explicitly show that $2 \mathrm{PI}$ WT identities constrain the UV divergences appearing at any finite approximation order in such a way that they can all be absorbed in the set of counterterms discussed here.

We stress again that the profusion of counterterms encountered here is a generic feature of 2PI renormalization theory and is a mere artifact of the finite approximation: different counterterms are nothing but different approximations of the true counterterms of the theory. For instance, the difference $\delta Z_{3}-\delta \bar{Z}_{3}$, or the gauge-fixing-parameter counterterm $\delta \bar{Z}_{4}=\delta \bar{\lambda} / \lambda_{R}$ should approach their exact (zero) value as the approximation order increases. As mentioned above, this is certainly true for the divergent parts of the respective counterterms. To ensure that this be true for the finite parts as well, one needs to impose suitable renormalization conditions. A related issue is the fact that, for a successful renormalization procedure, one needs to fix all the above counterterms without introducing any new input parameter - other than $m_{R}$ and $e_{R}$ - to specify the theory. 
In the 2PI formalism, the conditions which fix the various counterterms can be separated in two distinct classes. The first ones are the usual renormalization conditions, which define the theory. These can, for instance, be written in terms of the 2PI-resummed two- and three-point vertices $\Gamma_{R}^{(2)}$ and $\Gamma_{R}^{(3)}$. To be more specific, we denote by $\Sigma_{R}^{\bar{\psi} \psi}$ and $\Sigma_{R}^{A A} \equiv \Pi_{R}$ the $2 \mathrm{PI}$-resummed fermion and photon self-energies, i.e. the non-vanishing components of $\Sigma_{R}=i \Gamma_{R}^{(2)}+\mathcal{G}_{0, R}^{-1}$, and by $\Gamma_{R}^{(2,1)} \equiv \Gamma_{R, A \psi \bar{\psi}}^{(3)}$ the fermion-photon threepoint vertex. QED can be defined 8 by the following set of independent renormalization conditions at a given renormalization point $*$ (chosen here to correspond to zero photon and on-shell fermion momenta $\sqrt{9}$ ):

$$
\left.\Sigma_{R}^{\bar{\psi} \psi}\right|_{*}=0 \quad,\left.\quad \frac{\partial \Sigma_{R}^{\bar{\psi} \psi}}{\partial \not p}\right|_{*}=0 \quad,\left.\quad \frac{d \Pi_{R}^{T}}{d k^{2}}\right|_{*}=0 \quad \text { and }\left.\quad \Gamma_{R, \mu}^{(2,1)}\right|_{*}=-e_{R} \gamma_{\mu}
$$

where $\Pi_{R}^{T}$ is defined as, see (16), $\Pi_{R}^{\mu \nu}(k)=\left(g^{\mu \nu}-k^{\mu} k^{\nu} / k^{2}\right) \Pi_{R}^{T}\left(k^{2}\right)$.

The second class of conditions are in fact consistency conditions, whose role is to restore the identity of the various vertex functions at the renormalization point. It is a necessary requirement in order to guarantee that the approximation scheme converges toward the correct theory. For instance, we demand that the 2PI fermion self energy satisfies

$$
\left.\bar{\Sigma} \bar{\psi} \psi\right|_{*}=\left.\Sigma_{R}^{\bar{\psi} \psi}\right|_{*} \text { and }\left.\frac{\partial \bar{\Sigma}_{R}^{\bar{\psi} \psi}}{\partial \not p}\right|_{*}=\left.\frac{\partial \Sigma_{R}^{\bar{\psi} \psi}}{\partial \not p}\right|_{*}
$$

Similarly, we impose, for the longitudinal and transverse part of the 2PI photon polarization tensor,

$$
\left.\bar{\Pi}_{R}^{L}\right|_{*}=0 \quad,\left.\quad \frac{d \bar{\Pi}_{R}^{L}}{d k^{2}}\right|_{*}=0 \quad \text { and }\left.\quad \frac{d \bar{\Pi}_{R}^{T}}{d k^{2}}\right|_{*}=\left.\frac{d \Pi_{R}^{T}}{d k^{2}}\right|_{*} .
$$

where the first two conditions follow from the fact that, at any approximation order, the 2PI-resummed photon polarization tensor $\Pi_{R}$ is transverse in momentum space, i.e. $\Pi_{R}^{L}\left(k^{2}\right)=0$, see (16). Similar conditions for the $2 \mathrm{PI}$ four-photon function allows one to fix the four-photon counterterms $\delta \bar{g}_{1}$ and $\delta \bar{g}_{2}$ [2]. Finally, we demand that the different definitions of the three-point vertex coincide at the renormalization point 10

$$
\left.\frac{\delta \bar{\Sigma}_{R}^{\bar{\psi} \psi}}{\delta A_{R}}\right|_{*}=\left.\frac{\delta \bar{\Sigma}_{R}^{\bar{\psi} A}}{\delta \psi_{R}}\right|_{*}=\left.\Gamma_{R}^{(2,1)}\right|_{*}
$$

In total, the conditions (24)-(27) allow one to fix all the counterterms introduced above. We stress that conditions (25)-(27) are imposed by the consistency of the approximation scheme. The only freedom one has lies in the four independent renormalization conditions (24), as expected for QED.

\footnotetext{
8 To be complete, this should be supplemented by renormalization conditions for composite operators.

9 For a discussion of more generic renormalization points, see 3

${ }^{10}$ Lorentz symmetry and charge-conjugation invariance imply that $\delta \bar{\Sigma}_{R}^{\bar{\psi} A} /\left.\delta \psi_{R}\right|_{*}=\delta \bar{\Sigma}_{R}^{A \psi} /\left.\delta \bar{\psi}_{R}\right|_{*}[3]$.
} 


\section{Conclusion and Outlook}

To summarize, we have proposed a consistent, gauge-invariant 2PI renormalization procedure for abelian gauge theories and have clarified a number of issues concerning (gauge) symmetries in the 2PI formalism. We believe this is an important step, which opens the way to reliable quantitative calculations in gauge theories using nonperturbative 2PI techniques. Possible applications include equilibrium and nonequilibrium dynamics. This work is also an important step toward the case of non-abelian gauge theories 912.

Here, we would like to comment about another important issue concerning gauge theories in the 2PI formalism, namely the possibility (or not) to define gauge-fixing independent quantities. The point is that physical observables computed from the 2PI effective action usually contain residual, spurious gauge-fixing dependences at finite approximation order11, see e.g. 10[11. No systematic procedure has been found so far to get rid of the latter. General results [10] show however that these gauge dependent contributions are parametrically suppressed in powers of the coupling, which indicates that they should at least be well under control at weak coupling. Moreover, the observed good convergence properties of 2PI approximations schemes, see e.g. [6], suggest that gauge-fixing dependences may be controlled beyond the perturbative region. This has recently been tested for QED in Ref. [13, where the thermodynamic pressure has been computed from the (renormalized) 2PI loop-expansion at two-loop order in the covariant gauge. The results indicate that gauge-fixing parameter dependences remain under control in a wide range of couplings and are comparable with renormalization scale dependences, another source of uncertainty inherent in such calculations. Moreover, the Landau gauge has been identified as the gauge minimizing both gauge-fixing parameter and renormalization scheme dependences.

\section{References}

[1] U. Reinosa, J. Serreau, JHEP 0711 (2007) 097 arXiv:0708.0971 [hep-th]].

[2] U. Reinosa, J. Serreau, JHEP 0607 (2006) 028; See also Nucl. Phys. A 785 (2007) 230.

[3] U. Reinosa, J. Serreau, in preparation.

[4] H. van Hees, J. Knoll, Phys. Rev. D 65 (2002) 025010; ibid. 105005; Phys. Rev. D 66 (2002) 025028; J.-P. Blaizot, E. Iancu, U. Reinosa, Phys. Lett. B 568 (2003) 160; Nucl. Phys. A 736 (2002) 149.

[5] J. Berges, S. Borsányi, U. Reinosa, J. Serreau, Annals Phys. 320 (2005) 344.

[6] J. Berges, S. Borsányi, U. Reinosa, J. Serreau, Phys. Rev. D71 (2005) 105004; J.-P. Blaizot, A. Ipp, A. Rebhan, U. Reinosa, Phys. Rev. D72 (2005) 125005.

[7] F. Cooper, B. Mihaila, J.F. Dawson, Phys. Rev. D 70 (2004) 105008; Phys. Rev. D 71 (2005) 096003; Yu. B. Ivanov, F. Riek, H. van Hees, J. Knoll, Phys. Rev. D 72 (2005) 036008;

A. Arrizabalaga, U. Reinosa, Nucl. Phys. A 785 (2007) 234.

[8] U. Reinosa, Nucl. Phys. A 772 (2006) 138. See also hep-ph/0510380

[9] E. A. Calzetta, Int. J. Theor. Phys. 43 (2004) 767.

[10] A. Arrizabalaga, J. Smit, Phys. Rev. D66 (2002) 065014; M.E. Carrington, G. Kunstatter, H. Zaraket, Eur. Phys. J. C42 (2005) 253.

[11] E. Mottola, in SEWM02, Ed. M.G. Schmidt, World Scientific, Singapore hep-ph/0304279.

\footnotetext{
$\overline{11}$ The fact that the (renormalized) 2PI-resummed effective action is gauge-invariant does not guarantee that observables obtained from it are gauge-fixing independent.
} 
[12] J. Berges, Phys. Rev. D 70 (2004) 105010.

[13] S. Borsányi and U. Reinosa, Phys. Lett. B 661 (2008) 88. 